\title{
Retinal toxicity of amphotericin B in vitrectomised versus non-vitrectomised eyes
}

\author{
JOHN BALDINGER,' BERNARD H DOFT,' STEPHEN A BURNS, \\ AND BRUCE JOHNSON ${ }^{2}$
}

From 'Retina-Vitreous Consultants and the Departments of Ophthalmology and ${ }^{2}$ Pathology, the University of Pittsburgh School of Medicine, and Eye and Ear Hospital, Pittsburgh, Pennsylvania, USA

SUMMARY The retinal toxicity of intravitreally administered amphotericin B was compared in nonvitrectomised versus vitrectomised rabbit eyes. Doses of 5 and $10 \mu \mathrm{g}$ in both groups resulted in transient vitritis but had no effect on electroretinograms. Histopathological examination four weeks after injection showed vitreous cells and minimal areas of retinal necrosis in both groups at 5 or $10 \mu \mathrm{g}$ doses. At these doses vitrectomy did not modify the retinotoxic effects of intravitreally administered amphotericin B. At higher doses marked toxicity was found in both vitrectomised and non-vitrectomised groups.

The administration of amphotericin $B$ by direct intravitreal injection is accepted treatment in the management of fungal endophthalmitis, because the drug does not penetrate the vitreous cavity by other modes of administration. ${ }^{1-6}$ It is known that retinal toxicity may occur after intravitreal injection of amphotericin $B$ in the rabbit eye. ${ }^{78}$ Pars plana vitrectomy has also been employed in the management of fungal endophthalmitis. ${ }^{3-6}$ However, it is not yet known whether vitrectomy affects the retinal toxicity which may occur after intravitreal amphotericin B injection.

It is possible that vitrectomy could influence the toxicity of amphotericin B injected into the vitreous cavity in different ways. On one hand the removal of vitreous would remove a site for drug binding, and could allow easier drug passage to the retina, allowing one to postulate possible increase in toxicity. On the other hand the disappearance of amphotericin $B$ in vitrectomised rabbit eyes is accelerated in comparison with non-vitrectomised eyes. ${ }^{9}$ This would leave less drug in the vitreous cavity to interact with the retina, allowing one to postulate that vitrectomy might result in less retinal toxicity.

The purpose of this study was to evaluate in an animal model whether pars plana vitrectomy has an effect on the ocular toxicity of amphotericin B administered by direct intravitreal injection.

Correspondence to Bernard H Doft, MD, Retina-Vitreous Consultants, Eye and Ear Hospital, 230 Lothrop Street, Pittsburgh, PA 15213, USA.

\section{Material and methods}

New Zealand albino rabbits weighing 2.5 to $3.5 \mathrm{~kg}$ were employed. Anaesthesia was obtained with intramuscularly administered ketamine $(30 \mathrm{mg} / \mathrm{kg}$ of body weight) and xylazine ( $5 \mathrm{mg} / \mathrm{kg}$ of body weight), $2.0 \mathrm{ml}$ of retrobulbar $2 \%$ lignocaine, and topical $0.5 \%$ proxymetacaine (proparacaine). Cyclopentolate and phenylephrine were used for mydriasis.

Two groups of rabbits were prepared. (1) Vitrectomised. Animals underwent pars plana vitrectomy and lensectomy as described previously," with some modifications. A bent 20 -gauge needle was used for infusion during the lensectomy rather than a sutured infusion cannula. Infusion during vitrectomy was accomplished with a Charles infusion sleeve over the 20-gauge suction cutter (Ocutome). A transvitreal endoilluminator was employed. To separate the effects of possible vitrectomy-induced complications from toxic effect due to the drug, animals developing retinal detachments or other pathology during the two weeks after vitrectomy surgery were discarded before entry into the study (two animals). (2) Nonvitrectomised. These animals underwent no surgical manipulation.

Amphotericin B (Fungizone) was obtained from a single lot and reconstituted in sterile water immediately prior to use. Within each group of 16 animals four were assigned to intravitreal injection of one of four amphotericin B doses: 5, 10, 50, or $250 \mu \mathrm{g}$ (in a volume of $0.1 \mathrm{ml}$ ). Intravitreal injections were 
performed as previously described.' Each animal's paired eye served as a control, receiving the appropriate manipulation (vitrectomy/lensectomy versus non-vitrectomy) for that group, and $0.1 \mathrm{ml}$ of intravitreal balanced salt solution instead of amphotericin. After the injection all eyes were examined by ophthalmoscopy daily during weeks 1 and 2, and then every other day for weeks 3 and 4 .

Scotopic electroretinograms were obtained in both groups. The rabbits were preadapted to a background illumination of $3.9 \times 10^{-3}$ foot-lamberts for 5 minutes, followed by 12 minutes of dark adaptation. A Burian-Allen contact lens electrode was applied to the cornea, and a ground electrode was placed subdermally in the scalp. An integrated Ganzfeld flash stimulus of 1.07 foot-lambert-seconds was then used, each eye receiving five flashes (Grass PS-2 Photostimulator) at one-minute intervals. Electroretinograms were recorded with internally calibrated preamplifiers (Grass P-511) and a microcomputer. Responses were recorded on a floppy disc.

The A-B ratios were expressed as the ratio of the A-B amplitude (from the trough of the A wave to the peak of the $B$ wave) of the experimental eye to the A$B$ amplitude of the control eye. Vitrectomised animals had electroretinograms performed before vitrectomy, two weeks after vitrectomy but before intravitreal injection, and four weeks after intravitreal injection. Non-vitrectomised animals had electroretinograms done before intravitreal injection, two weeks after injection, and four weeks after injection. Both eyes of each animal were tested during the same session. ${ }^{11} 12$

Four weeks after amphotericin B injection the animals were killed and both eyes from all animals were enucleated and placed in formalin. Each eye was examined grossly. Routine histological sections were obtained for microscopic examination after the eye was embedded in paraffin. All histological sections were stained with haematoxylin and eosin and were read in masked fashion by an ophthalmic pathologist.

\section{Results}

\section{OCULAR EXAMINATIONS}

Non-vitrectomised eyes injected with 5 and $10 \mu \mathrm{g}$ amphotericin B showed no abnormalities except that in six of eight eyes there was mild vitreous haze. This cleared within one week. At $50 \mu \mathrm{g}$ all eyes developed a moderate vitreous haze persisting for a period of two to three weeks after injection. At $250 \mu \mathrm{g}$ severe vitreous haze persisting two to three weeks along with moderately dense cataracts developed in three of four eyes. All non-vitrectomised eyes at the 50 and $250 \mu \mathrm{g}$ dose also developed rhegmatogenous retinal

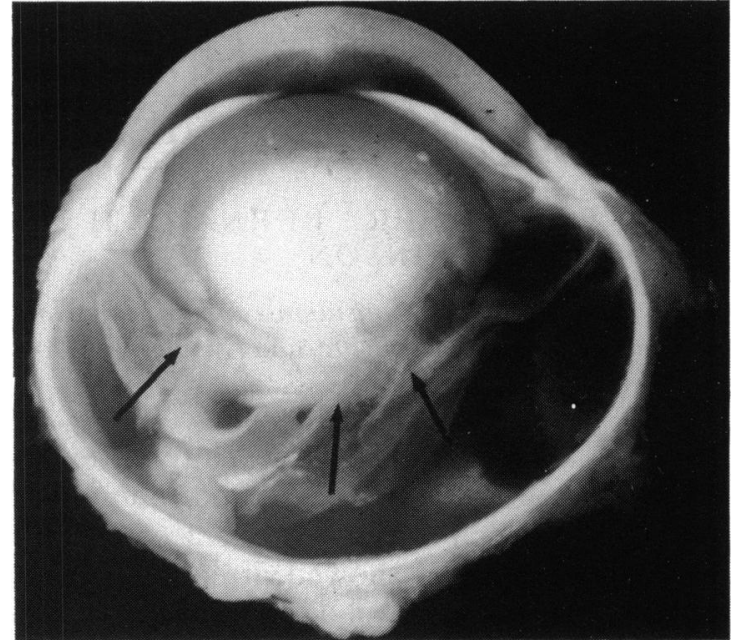

Fig. 1 Gross section from non-vitrectomised eye four weeks after $250 \mu \mathrm{g}$ intravitreal amphotericin injection. Note total retinal detachment (arrows), and subretinal haemorrhage.

detachments within two weeks of amphotericin B injection (Fig. 1).

The ophthalmological findings in the vitrectomised eyes injected with amphotericin B were similar to those of non-vitrectomised eyes. Mild transient vitreous haze was seen in five of eight eyes at the lower doses $(5$ and $10 \mu \mathrm{g})$. Rhegmatogenous retinal detachments developed in all eyes at the 50 and 250 $\mu \mathrm{g}$ doses. Interestingly, at the higher doses (50 and $250 \mu \mathrm{g}$ ) vitrectomised eyes were noted to have white fibrous-appearing strands within the vitreous cavity. This finding was not evident in non-vitrectomised eyes injected with amphotericin $B$ at the same doses.

Matched control eyes (which received intravitreal balanced salt solution) from both vitrectomised and non-vitrectomised groups did not show any pathological lesions.

\section{ELECTRORETINOGRAPHY}

The results of electroretinography are shown in Table 1. The mean A-B ratios of the 5 and $10 \mu \mathrm{g}$ vitrectomised and non-vitrectomised eyes were similar (with overlap of data points at each concentration), and there was no statistical difference between the groups. At higher drug doses $(50 \mu \mathrm{g}$ and $250 \mu \mathrm{g}$ ), where retinal detachment occurred in all eyes, the groups did differ, since all except one of the vitrectomised eyes had extinguished electroretinograms (Table 1). This effect of vitrectomy at high dose was significant ( $p<0 \cdot 01$, Fisher's exact test).

A-B amplitude ratios in vitrectomised animals two weeks after vitrectomy but prior to injection were virtually identical when compared with the same 
Table 1 Ratio of the mean amphotericin injected $A-B$ amplitude to control $A-B$ amplitude

\begin{tabular}{llc}
\hline Dose (micrograms) & Non-vitrectomised & Vitrectomised \\
\hline 5 & $0 \cdot 87(0 \cdot 11)$ & $0.94(0 \cdot 08)$ \\
10 & $1 \cdot 04(0 \cdot 17)$ & $1 \cdot 06(0 \cdot 15)$ \\
50 & $0 \cdot 41(0 \cdot 21)$ & $<0 \cdot 01(-)^{*}$ \\
250 & $0 \cdot 19(0 \cdot 18)$ & $0 \cdot 01(0 \cdot 25)^{*}$ \\
\hline
\end{tabular}

*The 50 and $250 \mu \mathrm{g}$ vitrectomised eyes had A-B ratios approaching zero. To ascertain whether there was any electroretinographic activity at all in these eyes we also used longer adaptation times and increased stimulus intensities. These manipulations did not result in increased responses.

animal's ratio determined before vitrectomy. This indicates that surgery itself did not have an adverse effect on the electroretinogram.

\section{HISTOPATHOLOGY}

In both groups at lower drug doses $(5 \mu \mathrm{g}$ and $10 \mu \mathrm{g})$ mild vitreous inflammation with an increase of polymorphonuclear leucocytes and lymphocytes was seen (Table 2). Segmental microscopic retinal necrosis was also noted in lower dose vitrectomised and nonvitrectomised eyes (Fig. 2). More extensive retinal necrosis was observed at the 50 and $250 \mu \mathrm{g}$ concentrations in both groups (Fig. 3). Some of the 50 and $250 \mu \mathrm{g}$ treated eyes in both groups showed choroiditis
Table 2 Histopathology four weeks after intravitreal amphotericin $B$ injection

\begin{tabular}{|c|c|c|c|c|c|c|c|c|}
\hline & \multicolumn{8}{|c|}{ Amphotericin dose } \\
\hline & \multicolumn{2}{|c|}{$5 \mu g$} & \multicolumn{2}{|c|}{$10 \mu g$} & \multicolumn{2}{|c|}{$50 \mu g$} & \multicolumn{2}{|c|}{$250 \mu g$} \\
\hline & $N V$ & $V$ & $N V$ & $\boldsymbol{V}$ & $N V$ & $V$ & $N V$ & $V$ \\
\hline Number of eyes & 4 & 4 & 4 & 4 & 4 & 4 & 4 & 4 \\
\hline Vitritis & 3 & 2 & 3 & 3 & 4 & 3 & 4 & 3 \\
\hline Choroiditis & 0 & 0 & 0 & 0 & 2 & 2 & 3 & 2 \\
\hline Retinal detachment & 0 & 0 & 0 & 0 & 4 & 4 & 4 & 4 \\
\hline $\begin{array}{l}\text { Microscopic retinal } \\
\text { necrosis }\end{array}$ & 3 & 3 & 3 & 3 & 4 & 4 & 4 & 4 \\
\hline Cyclitic membrane & 0 & 0 & 0 & 0 & 0 & 0 & 1 & 0 \\
\hline
\end{tabular}

$\mathrm{V}=$ vitrectomised eye $\mathbf{N V}=$ non-vitrectomised eye .

with a predominately lymphocytic infiltrate (Table 2). Retinal detachments were noted in all eyes of both groups receiving 50 or $250 \mu \mathrm{g}$ (Fig. 1). Prominent fibrogliotic periretinal tissue was noted histopathologically in the higher dose (50 and 250 $\mu \mathrm{g}$ ) vitrectomised group eyes (Fig. 4), but not in the similarly treated non-vitrectomised eyes. Fibrogliotic elements were sometimes noted to exert tractional forces on the retina. At histopathological examination only the amphotericin treated vitrectomised eyes showed periretinal proliferations.

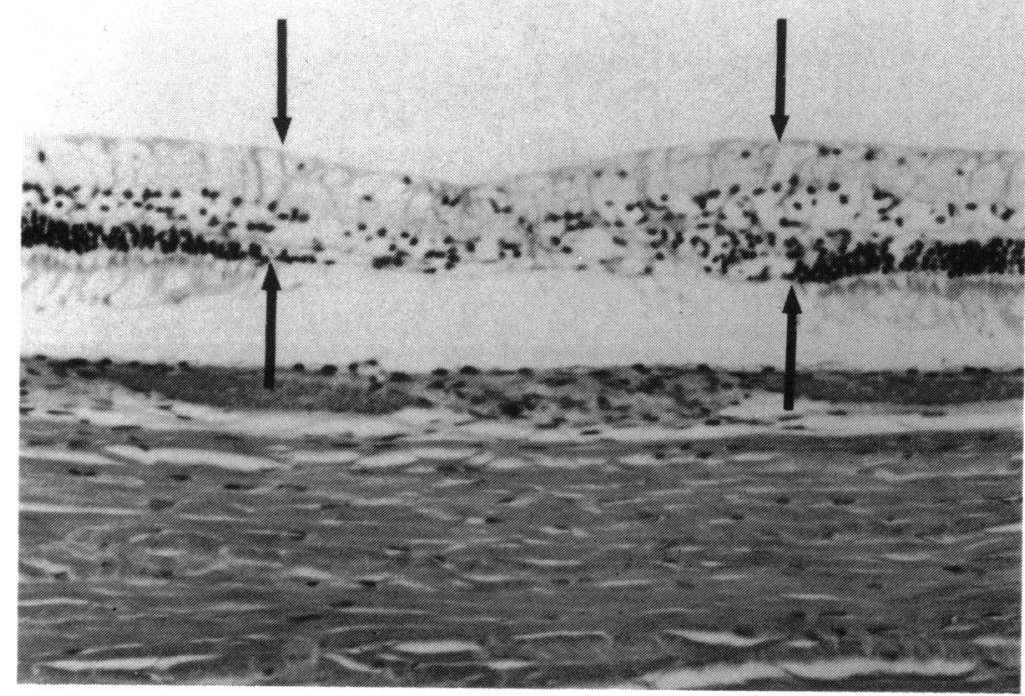

Fig. 2 Segmental area of retinal necrosis (between arrows) with normal adjacent retina, seen in $5 \mu \mathrm{g}$ non-vitrectomised eye four weeks after injection of amphotericin. Similar findings were noted in both 5 and $10 \mu \mathrm{g}$ vitrectomised and nonvitrectomised groups. Retinal detachment is artefactual. (Haematoxylin-eosin, $\times 350$ ). 
Fig. 3 More extensive full thickness retinal necrosis with normal adjacent retina seen in 50 $\mu \mathrm{g}$ non-vitrectomised eyes four weeks after injection. Similar findings noted in both 50 and $250 \mu \mathrm{g}$ non-vitrectomised and vitrectomised eyes. (Haematoxylineosin, $\times 200$ ).

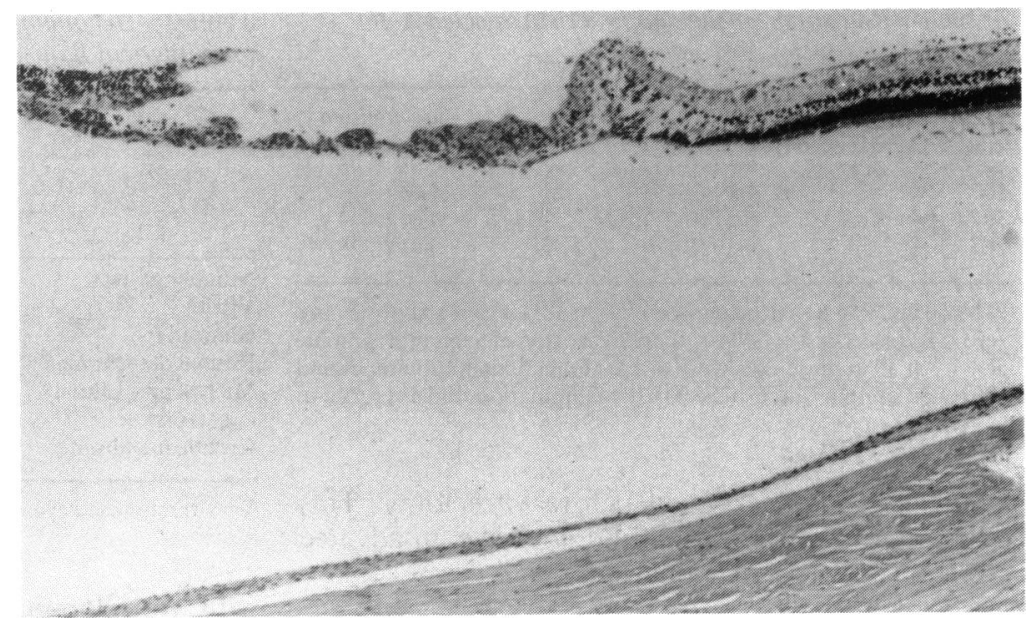

\section{Discussion}

Although the use of intravitreal antibiotics is becoming an accepted form of treatment for endophthalmitis, the role of pars plana vitrectomy in the therapy

of these infections is not clearly defined. Therapeutic vitrectomy seems logical, allowing incision and drainage to remove the bulk of infectious organisms and associated inflammatory debris. Cottingham and Forster ${ }^{13}$ have shown in a rabbit model of bacterial

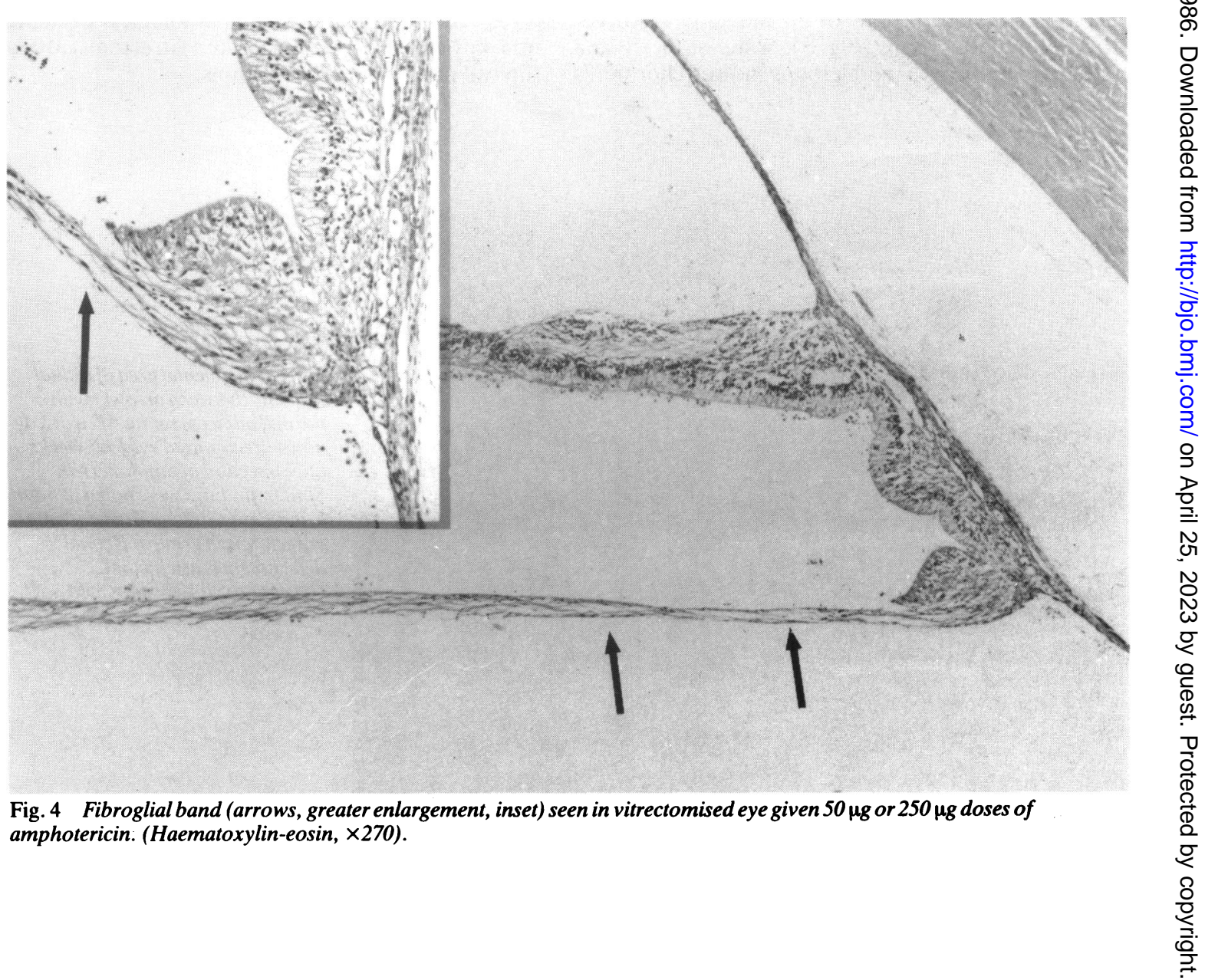


endophthalmitis that eyes treated with combined vitrectomy and intraocular antibiotics had a greater number of negative cultures at one week than if treated with intraocular antibiotics alone.

Previous studies have been performed to assess retinal toxicity after direct intravitreal injection of amphotericin in the rabbit model. However, to our knowledge data from controlled studies regarding retinal toxicity in vitrectomised compared with nonvitrectomised eyes has not previously been reported. One study has looked at the effect of intravitreally administered amphotericin B after vitrectomy, but that study did not employ non-vitrectomised controls. ${ }^{14}$ That study did not demonstrate toxicity of intravitreal amphotericin at doses of $5 \mu \mathrm{g}$ or less. Another study looked at the toxicity of amphotericin B methyl ester administered in vitrectomy infusion fluid. ${ }^{15}$ In that study the maximum non-toxic dose of the methyl ester in the vitreous infusion fluid was 75 $\mu \mathrm{g} / \mathrm{ml}$. However, that is not analogous to regimens in which vitrectomy is followed by isolated intravitreal amphotericin B injections.

A previous study concerning clearance of amphotericin after intravitreal injection shows that eyes that had undergone vitrectomy/lensectomy cleared amphotericin much more rapidly than non-vitrectomised eyes or eyes which had had lensectomy alone."

Our data indicate that only minimal retinal toxicity develops at 5 and $10 \mu \mathrm{g}$ doses of amphotericin in both vitrectomised and non-vitrectomised eyes. Except for transient vitritis and small skip areas of retinal necrosis seen histopathologically, these eyes appeared normal, and electroretinogram A-B amplitude ratios were unaffected. Thus vitrectomy does not appear to increase the degree of retinal toxicity from amphotericin at doses employed clinically. However, at the 50 and $250 \mu \mathrm{g}$ concentrations marked retinal toxicity was apparent clinically, electroretinographically, and histopathologically in both vitrectomised and non-vitrectomised eyes. At these highly toxic dose levels electroretinogram effects were found to be more marked in vitrectomised eyes than in non-vitrectomised eyes. We are unable to explain this statistically significant finding, since retinal detachments were present in all eyes of both groups at these dose levels.
The data presented in this study demonstrate that vitrectomy makes the eye neither more susceptible nor less susceptible to toxic effects of intravitreal amphotericin at doses commonly employed clinically. However, the extent to which these data obtained in an animal model applies to humans is unknown.

This work was supported in part by grants from the Pennsylvania Lions Sight Conservation and Eye Research Foundation, Inc, and the National Institutes of Health, R01-EY04395.

\section{References}

1 Stern GA, Fetkenhour CL, O'Grady RB. Intravitreal amphotericin B treatment of candida endophthalmitis. Arch Ophthalmol 1977; 95: 89-93.

2 Perraut LE Jr, Perraut LE, Bleiman B, Lyons J. Successful treatment of Candida albicans endophthalmitis with intravitreal amphotericin B. Arch Ophthalmol 1981; 99: 1565-7.

3 Boldrey EE. Bilateral endogenous aspergillus endophthalmitis. Retina 1981; 1: 171-4.

4 Gilbert CM, Novak MA. Successful treatment of postoperative candida endophthalmitis in an eye with an intraocular lens implant. Am J Ophthalmol 1984; 97: 593-5.

5 Ho PC, Tolentino FJ, Baker AS. Successful treatment of exogenous aspergillus endophthalmitis: a case report. $\mathrm{Br} J$ Ophthalmol 1984; 68: 412-5.

6 Peyman GA, Vastine GW, Diamond JG. Vitrectomy in exogenous candida endophthalmitis. Graefes Arch Klin Exp Ophthalmol 1975; 197: 55-9.

7 Axelrod AJ, Peyman GA, Apple DJ. Toxicity of intravitreal injection of amphotericin B. Am J Ophthalmol 1973; 76: 578-83.

8 Souri EN, Green WR. Intravitreal amphotericin B toxicity. Am J Ophthalmol 1974; 78: 77-81.

9 Doft B, Weiskopf J, Nilsson-Ehle I, Wingard LB. Amphotericin clearance in vitrectomized versus non-vitrectomized eyes. Ophthalmology (Rochester) in press.

10 Stern WH, Fisher SK, Anderson DH, et al. Epiretinal membrane formation after vitrectomy. Am J Ophthalmol 1982; 93: 75772.

11 Lawwill T. Practical rabbit electroretinography. Am J Ophthalmol 1972; 74: 135-41.

12 Spivey BE, Pearlman JT. Day-to-day variations in the ERG of humans and rabbits. Am J Ophthalmol 1963; 55: 1013-20.

13 Cottingham AJ, Forster RK. Vitrectomy in endophthalmitis. Arch Ophthalmol 1976; 94: 2078-81.

14 Huang K, Peyman GA, McGetriele J. Vitrectomy in experimental endophthalmitis in fungal infection. Ophthalmic Surg 1979; 10: 84-6.

15 Raichand M, Peyman GA, West SC, Hammond G, Zweig K. Toxicity and efficacy of vitrectomy fluids: amphotericin $B$ methyl ester in the treatment of experimental fungal endophthalmitis. Ophthalmic Surg 1980; 11: 246-8.

Accepted for publication 16 December 1985. 